1 Universidade Federal do Triângulo Mineiro (UFTM) Departamento de Terapia Ocupacional - Uberaba (MG), Brasil.

erikatouftm@hotmail.com

2 Universidade Federal do Triângulo Mineiro (UFTM), Departamento de Saúde Coletiva - Uberaba (MG), Brasil.

\section{Centros de Atenção Psicossocial - álcool e drogas: perfil dos usuários}

\author{
Psychosocial Care Centers - alcohol and drugs: users' profile
}

Erika Renata Trevisan', Sybelle de Souza Castro ${ }^{2}$

DOI: 10.1590/0103-1104201912113

RESUMO O estudo objetivou descrever o perfil dos usuários em tratamento nos Centros de Atenção Psicossocial - álcool e drogas (CAPSad) da região do Triângulo Mineiro, em Minas Gerais. Estudo de tipo transversal, com amostra de 369 usuários. Utilizou-se um instrumento contendo variáveis sociodemográficas e sobre o atendimento no CAPSad; sobre substâncias psicoativas utilizadas ao longo da vida e no último mês; idade de uso inicial; internação e medicação decorrentes do uso; tempo e modalidade de tratamento. Realizaram-se análise estatística descritiva e testes t-Student, não paramétrico de Mann-Whitney, análise de variância paramétrica (ANOVA-F), de comparações múltiplas de Dunn. Verificaram-se maiores percentuais de homens (80,5\%), com idade entre 41 e 60 anos (45,8\%), sem companheiro(a) (55,2\%), com ensino fundamental incompleto (38,4\%), desempregados (34,7\%) e em tratamento entre 1 e 5 anos (62,9\%). O álcool foi a substância mais utilizada ao longo da vida (89,7\%); e no último mês (77,8\%). Este estudo reforça a necessidade de resgate da autonomia, da reinserção social, do trabalho com valor social e dos direitos de cidadania, a partir de uma atenção especializada e individualizada. As redes sociais de apoio, a intersetorialidade e a articulação das Redes de Atenção Psicossocial devem ser a base da transformação para que essas questões se concretizem na prática assistencial.

PALAVRAS-CHAVE Saúde mental. Epidemiologia. Pesquisa sobre serviços de saúde. Serviços de saúde mental. Transtornos relacionados ao uso de substâncias.

ABSTRACT The study aimed to describe the profile of users being treated in the Psychosocial Care Centers - alcohol and drugs (CAPSad) of the Triângulo Mineiro region of Minas Gerais. A cross-sectional study, with sample of 369 users into treatment. An instrument was used containing sociodemographic variables and about care in CAPSad; about psychoactive substances used throughout life and in the last month; age of initial use of psychoactive substances; hospitalization and medication resulting from the use; time and modality of treatment. Descriptive statistical analysis and Student's t-tests, non-parametric Mann-Whitney, parametric analysis of variance (ANOVA-F), and Dunn's multiple comparisons were performed. There was a higher percentage of men (80.5\%), age between 41 and 60 years (45.8\%), without partner (55.2\%), incomplete elementary school (38.4\%), unemployed (34.7\%) and in treatment between 1 and 5 years (62.9\%). Alcohol was the most used substance throughout life (89.7\%); and in the last month (77.8\%). This study reinforces the need to rescue autonomy, social reintegration, work with social value, citizenship rights based on specialized and individualized attention. Social support networks, intersectoriality and 
the articulation of the Psychosocial Attention Networks should be the basis of the transformation so that these issues are concretized in the care practice.

KEYWORDS Mental health. Epidemiology. Health services research. Mental health services. Substance-related disorders.

\section{Introdução}

As políticas atuais de saúde mental e de atenção psicossocial desenvolvidas no âmbito do Sistema Único de Saúde (SUS), resultantes do movimento da reforma psiquiátrica, têm uma relação direta com o movimento da reforma sanitária, que surgiu em meados da década de 1970 e constituiu-se no processo de amplas mobilizações da sociedade brasileira pela redemocratização'.

Esse processo sociopolítico possibilitou diferentes e intensas transformações na atenção à saúde mental nas últimas três décadas. $\mathrm{O}$ movimento de reforma psiquiátrica consolidou mudanças significativas na forma de tratar e entender os transtornos mentais, que passaram a ser cuidados nos serviços substitutivos ao hospital psiquiátrico e na superação do modelo psiquiátrico, pautado na exclusão social, na violência e na cronificação do sujeito ${ }^{2}$.

Diversas estratégias foram desenvolvidas nos campos técnico, assistencial, social, político e jurídico, e, em 2011, foi instituída a Rede de Atenção Psicossocial (Raps) a partir da Portaria GM/MS n 3.088 , de dezembro de 2011, que possibilita uma nova dimensão ao conjunto das ações em saúde mental no SUS, com a garantia da articulação e da integração dos serviços de atenção das redes de saúde no território, qualificando o cuidado por meio do acolhimento, do acompanhamento contínuo e da atenção às urgências à atenção psicossocial da população com transtornos mentais e com necessidades decorrentes do uso do crack, álcool e outras drogas e de seus familiares, em seus diferentes níveis de complexidade'.

Na Raps, os Centros de Atenção Psicossocial (Caps) foram designados como locais de referência para o tratamento da população adulta com transtornos mentais severos e persistentes, os Centros de Atenção Psicossocial Infanto-Juvenil (CAPSi) são especializados em crianças e adolescentes com transtornos mentais, e os Centros de Atenção Psicossocial álcool e drogas (CAPSad) destinados às pessoas com problemas decorrentes do uso de álcool e outras drogas ${ }^{3}$.

Essa mudança de paradigma do cuidado das pessoas com problemas decorrentes do uso de álcool e outras drogas deve incluir a relação que se estabelece com o usuário, a equipe, a família e a comunidade. A mudança de papéis, a democratização das instituições, o envolvimento e a responsabilização da comunidade devem somar-se aos objetivos técnicos do tratamento. A partir disso, o objeto de intervenção torna-se mais complexo e interdisciplinar, assim como as práticas e os saberes tradicionais devem ser reconstruídos para responder às demandas dessa transformação ${ }^{4}$.

De acordo com o 'Relatório Mundial sobre Drogas', publicado em 2016 pelo Escritório das Nações Unidas sobre Drogas e Crime (UNODC), aproximadamente $5 \%$ da população adulta e 250 milhões de pessoas entre 15 e 64 anos usaram pelo menos uma droga em 2014. Com relação à mortalidade relacionada ao uso de drogas, 207 mil mortes foram relatadas, sendo um terço delas por 
overdose. O consumo de heroína e as mortes por overdose aumentaram nos últimos dois anos em alguns países da América do Norte, da Europa Ocidental e Central. Os opioides continuam a representar os maiores riscos de danos à saúde entre as principais drogas. Com exceção do álcool e do tabaco, a maconha é a droga mais usada ao redor do mundo. $\mathrm{O}$ acesso a serviços de tratamento, com base em evidências científicas, representa outro grande desafio apontado pelo relatório, pois somente uma em cada seis pessoas que necessitam de atendimento tem acesso aos serviços ${ }^{5}$.

No Brasil, aproximadamente $12,3 \%$ da população é considerada dependente de álcool, de acordo com os critérios da $10^{\mathrm{a}}$ Classificação Internacional de Doenças (CID-10) e do IV Manual Diagnóstico e Estatístico de Transtornos Mentais (DSMIV), sendo que a prevalência é de $17,1 \%$ entre a população masculina e $5,7 \%$ na população feminina, que são prevalências consideradas altas quando comparadas às de outras condições de saúde. Além dos problemas de saúde física, as pessoas com transtornos causados pelo uso de álcool e outras drogas podem ter sofrimentos psicológicos e psicossociais graves, problemas interpessoais, perda de emprego, dificuldades de aprendizado, além de problemas legais ${ }^{6}$.

Embora nem todo usuário de substâncias psicoativas deva ser considerado dependente, é preciso ressaltar que, de acordo com a Organização Mundial da Saúde (OMS), 10\% das populações dos centros urbanos de todo o mundo, incluindo o Brasil, necessitam de assistência integral à saúde para eliminar ou minimizar os danos que essas substâncias podem causar. Nesse sentido, os CAPSad são os serviços públicos de saúde preconizados para o tratamento dessa população no Brasil ${ }^{3}$.

Este estudo, portanto, teve como objetivo descrever o perfil epidemiológico e os tipos de substâncias psicoativas utilizadas pelos usuários dos CAPSad da região do Triângulo Mineiro, no estado de Minas Gerais.

\section{Métodos}

Trata-se de um estudo descritivo com abordagem quantitativa do tipo transversal. A pesquisa foi aprovada pelo Comitê de Ética em Pesquisa por meio da Plataforma Brasil, com parecer consubstanciado de número 1.139.451 e CAAE 45867315.9.0000.5154.

Os dados relativos aos clientes foram obtidos por meio dos prontuários, e a pesquisa foi realizada nos três CAPSad da região do Triângulo Mineiro, sendo que um deles é CAPSad II e dois são CAPSad III. Os CAPSad II atendem cidades e/ou regiões com pelo menos 70 mil habitantes durante o dia. Os CAPSad III possuem atendimento noturno e observação, funcionam 24 horas e atendem cidades e ou regiões com pelo menos 150 mil habitantes.

A população assistida por esses serviços totaliza 920.033 habitantes em duas macrorregiões de saúde do estado de Minas Gerais: Triângulo Norte e Triângulo Sul.

No período da coleta dos dados, foi realizado um levantamento dos clientes que estavam em atendimento nos CAPSad, para a realização do cálculo e do sorteio da amostra. O sorteio foi realizado no Programa SPSS-versão 21. O cálculo do tamanho amostral considerou uma prevalência de uso de múltiplas substâncias psicoativas de $39,0 \%$, uma precisão de $4 \%$ e um intervalo de confiança de $95 \%$. Para uma população finita de 963 clientes dos serviços, obteve-se o tamanho amostral de 369 participantes a serem incluídos no estudo. A coleta dos dados foi realizada no período de maio a agosto de 2016.

Foi desenvolvido um instrumento para a coleta de dados com as seguintes variáveis: sexo; idade; estado civil; escolaridade; situação de trabalho; demanda do atendimento no CAPSad; substâncias psicoativas utilizadas ao longo da vida e no último mês; idade de uso inicial das substâncias psicoativas; internação e medicação decorrentes do uso de substâncias psicoativas; tempo de permanência no CAPSad e modalidade de tratamento. Inicialmente, constavam do instrumento variáveis como: 
renda, religião, problemas psicossociais e comorbidades relacionadas ao uso de substâncias, entretanto, essas informações não foram encontradas na maioria dos prontuários investigados, não sendo possível a análise desses dados.

Para a análise dos dados, foi utilizado o teste t-Student, quando as variáveis epidemiológicas e sociodemográficas tinham apenas duas categorias e os dados apresentassem normalidade. Quando a normalidade não foi satisfeita, foi utilizado o teste não paramétrico de Mann-Whitney. Quando apresentaram mais de duas categorias nas variáveis epidemiológicas e sociodemográficas, os dados apresentaram normalidade e as variâncias foram homogêneas, foi utilizada a análise de variância paramétrica (ANOVA-F), seguida do teste de comparação múltipla de Tukey. Quando a normalidade dos dados e a homogeneidade das variâncias não foram satisfeitas, a ANOVA não paramétrica foi usada pelo teste de Kruskal-Wallis, seguido do teste de comparações múltiplas de Dunn. O nível de significância para todos testes foi de $5 \%$.

\section{Resultados}

Verificou-se maior percentual do sexo masculino (80,5\%), entre 41 e 60 anos (45,8\%) e sem companheiro(a) $(55,2 \%)$. A maioria possui ensino fundamental incompleto $(38,4 \%)$ e está desempregada (34,7\%) (tabela 1).

Tabela 1. Perfil sociodemográfico dos clientes atendidos nos CAPSad da Região do Triângulo Mineiro (MG), Brasil, 2016

\begin{tabular}{|c|c|c|}
\hline \multirow[t]{2}{*}{ Variáveis/Categorias } & \multicolumn{2}{|c|}{ Distribuição } \\
\hline & $(n=369)$ & $\%$ \\
\hline \multicolumn{3}{|l|}{ Sexo } \\
\hline Masculino & 297 & 80,5 \\
\hline Feminino & 67 & 18,2 \\
\hline Não informado & 5 & 1,3 \\
\hline \multicolumn{3}{|l|}{ Idade (em anos) } \\
\hline Até 20 anos & 8 & 2,2 \\
\hline De 21 a 40 anos & 149 & 40,4 \\
\hline De 41 a 60 anos & 169 & 45,8 \\
\hline Acima de 61 anos & 34 & 9,2 \\
\hline Não informado & 9 & 2,4 \\
\hline \multicolumn{3}{|l|}{ Estado civil } \\
\hline Solteiro & 147 & 39,8 \\
\hline Casado & 91 & 24,7 \\
\hline União estável & 46 & 12,5 \\
\hline Viúvo & 9 & 2,4 \\
\hline Divorciado & 48 & 13,0 \\
\hline Não informado & 28 & 7,6 \\
\hline \multicolumn{3}{|l|}{ Escolaridade } \\
\hline Analfabeto & 9 & 2,4 \\
\hline Ensino fundamental I incompleto & 71 & 19,2 \\
\hline Ensino fundamental I completo & 30 & 8,1 \\
\hline
\end{tabular}




\begin{tabular}{lrr} 
Tabela 1. (cont.) & 71 & 19,2 \\
\hline Ensino fundamental II incompleto & 45 & 12,2 \\
Ensino fundamental II completo & 27 & 7,3 \\
Ensino médio incompleto & 42 & 11,4 \\
Ensino médio completo & 8 & 2,2 \\
Ensino superior incompleto & 3 & 0,9 \\
Ensino superior completo & 63 & 17,1 \\
Não informado & 67 & 18,2 \\
\hline Tipo de ocupação & 1 & 0,3 \\
\hline Carteira assinada & 51 & 13,8 \\
Cooperativa & 128 & 34,7 \\
Autônomo & 15 & 4,1 \\
Desempregado & 3 & 0,8 \\
Aposentado por tempo de trabalho & 104 & 28,2 \\
Aposentado por invalidez & & \\
Não informado & 3 & \\
\hline
\end{tabular}

Fonte: Banco de dados da pesquisa sobre o perfil dos clientes dos CAPSad da região do Triângulo Mineiro (MG), 2016.

A maioria dos clientes está em tratamento entre 1 e 5 anos (62,9\%); houve maior percentual de usuários que chegaram até o CAPSad por meio de encaminhamentos (44,7\%), realizados principalmente pelas Unidades Básicas de Saúde (UBS) (6,0\%), seguidos de demanda espontânea do usuário ao serviço (43,7\%) (tabela 2).

Foi expressiva a quantidade de usuários dos
CAPSad que já haviam sido internados por uso de substâncias psicoativas (39,8\%), entretanto, não constava tal informação nos prontuários para $26,3 \%$ dos participantes do estudo. Faziam uso de medicações relacionadas aos problemas decorrentes do uso das substâncias 64,8\%, e a modalidade de tratamento mais utilizada nos CAPSad foi a semi-intensiva (61,0\%) (tabela 2).

Tabela 2. Perfil dos clientes atendidos nos CAPSad, segundo tempo de tratamento, demanda pelo atendimento, formas de encaminhamento, modalidade de tratamento e se houve internação. Triângulo Mineiro (MG), Brasil, 2016

\begin{tabular}{lrr}
\hline Variáveis/Categorias & \multicolumn{2}{c}{ Distribuição } \\
\cline { 2 - 3 } & $(\mathbf{n = 3 6 9 )}$ & $\%$ \\
\hline Tempo de tratamento no CAPSad (em anos) & & 7,0 \\
\hline Menos de 1 ano & 26 & 62,9 \\
De 1 a 5 anos & 83 & 22,5 \\
De 5 a 10 anos & 28 & 7,6 \\
$\geq 10$ anos & & 43,7 \\
\hline Demanda pelo atendimento & 161 & 44,7 \\
\hline Busca espontânea & 165 & 2,2 \\
Encaminhamento & 8 & \\
Busca da família & & \\
\hline
\end{tabular}




\begin{tabular}{|c|c|c|}
\hline Busca de amigos & 2 & 0,5 \\
\hline Ordem judicial & 3 & 0,8 \\
\hline Trabalho & 1 & 0,3 \\
\hline Não informado & 29 & 7,9 \\
\hline \multicolumn{3}{|l|}{ Formas de Encaminhamento } \\
\hline Unidade Básica de Saúde (UBS) & 22 & 6,0 \\
\hline Programa de Saúde da Família (PSF) & 10 & 2,7 \\
\hline Unidades de emergência & 4 & 1,1 \\
\hline Centros de Referência de Assistência Social (Cras) & 2 & 0,5 \\
\hline Grupos de apoio & 1 & 0,3 \\
\hline Hospital psiquiátrico & 20 & 5,4 \\
\hline Hospital geral & 12 & 3,3 \\
\hline Ordem judicial & 15 & 4,1 \\
\hline Outros & 83 & 22,5 \\
\hline Não se aplica & 170 & 46,1 \\
\hline Não informado & 30 & 8,1 \\
\hline \multicolumn{3}{|l|}{ Internação por uso de substância psicoativa } \\
\hline Sim & 147 & 39,8 \\
\hline Não & 125 & 33,9 \\
\hline Não informado & 97 & 26,3 \\
\hline \multicolumn{3}{|c|}{ Faz uso de medicação devido ao uso de substância psicoativa } \\
\hline $\operatorname{Sim}$ & 239 & 64,8 \\
\hline Não & 79 & 21,4 \\
\hline Não informado & 51 & 13,8 \\
\hline \multicolumn{3}{|l|}{ Modalidade de atendimento no CAPSad } \\
\hline Intensivo & 68 & 18,4 \\
\hline Semi-intensivo & 225 & 61,0 \\
\hline Não intensivo & 42 & 11,4 \\
\hline Não informado & 34 & 9,2 \\
\hline
\end{tabular}

Fonte: Banco de dados da pesquisa sobre o perfil dos clientes dos CAPSad da região do Triângulo Mineiro (MG), 2016.

A tabela 3 apresenta os tipos de drogas consumidas durante a vida: $89,7 \%$ consumiram álcool ao longo da vida, seguido pelo tabaco (58,3\%), enquanto as substâncias alucinógenas e o LSD foram os menos consumidos $(2,2 \% \mathrm{e}$
0,5\%), respectivamente. Apresenta, também, o consumo no último mês, tendo como referência o momento do acolhimento no CAPSad. O álcool foi a substância mais utilizada (77,8\%); e o LSD e o êxtase foram as menos ( $0 \%$ e $0,3 \%$ ). 
Tabela 3. Drogas utilizadas durante a vida e no último mês pelos clientes dos CAPSad do Triângulo Mineiro (MG), Brasil, $2016(N=365)$

\begin{tabular}{lcccr}
\hline Drogas utilizadas $^{\star}$ & Uso na vida & \multicolumn{3}{c}{ Uso no mês } \\
\cline { 2 - 5 } & $\mathbf{n}$ & \% & $\mathbf{n}$ & \% \\
\hline Tabaco & 215 & 58,3 & 160 & 43,4 \\
Álcool & 331 & 89,7 & 287 & 77,8 \\
Maconha & 166 & 45,0 & 97 & 26,3 \\
Cocaína & 141 & 38,2 & 72 & 19,5 \\
Crack & 161 & 43,6 & 124 & 33,6 \\
Anfetamina & 9 & 2,4 & 4 & 1,1 \\
Benzodiazepínico & 11 & 3,0 & 5 & 1,4 \\
Solvente & 15 & 4,1 & 4 & 1,1 \\
LSD & 2 & 0,5 & 0 & 0 \\
Alucinógenos & 8 & 2,2 & 4 & 1,1 \\
Extase & 2 & 0,5 & 1 & 0,3 \\
Outras & 16 & 4,3 & 7 & 1,9 \\
\hline
\end{tabular}

Fonte: Banco de dados da pesquisa sobre o perfil dos clientes dos CAPSad da região do Triângulo Mineiro (MG), 2016.

* Não informaram $(n=4)$.

Nota: Categorias não são mutuamente exclusivas.

Verificou-se que a droga mais consumida entre os homens foi o tabaco $(81,3 \%)$, e entre as mulheres foi o crack (22,6\%). Com relação à faixa etária, a droga mais consumida entre os adultos $(77,4 \%)$ e os idosos $(83,8 \%)$ foi o álcool. Os usuários dos CAPSad que possuíam companheiro(a) consumiam mais álcool
(42,9\%), e os que não possuíam utilizavam a cocaína (71,0\%). Considerando a situação de trabalho, verificou-se que a droga mais consumida pelas pessoas que possuíam ocupação foi o álcool (44,7\%), e a mais consumida pelos que não possuíam ocupação foi o crack $(64,0 \%)$ (tabela 4).

Tabela 4. Drogas utilizadas no último mês, no momento do acolhimento nos CAPSad, segundo sexo, faixa etária, estado civil e situação de trabalho. Triângulo Mineiro (MG), Brasil, 2016

\begin{tabular}{|c|c|c|c|c|}
\hline \multirow[t]{3}{*}{ Tipos de Drogas } & \multicolumn{4}{|c|}{ Sexo $(n=364)$ - Não informaram $(n=5)$} \\
\hline & \multicolumn{2}{|c|}{ Homens (n=297 - 80,5\%) } & \multicolumn{2}{|c|}{ Mulheres $(n=67-18,2 \%)$} \\
\hline & $\mathrm{n}$ & $\%$ & $\mathbf{n}$ & $\%$ \\
\hline Tabaco & 130 & 81,3 & 28 & 17,5 \\
\hline Álcool & 232 & 80,9 & 50 & 17,4 \\
\hline Maconha & 78 & 80,4 & 17 & 17,5 \\
\hline Cocaína & 55 & 76,4 & 16 & 22,2 \\
\hline Crack & 94 & 75,8 & 28 & 22,6 \\
\hline
\end{tabular}


Tabela 4. (cont.)

\begin{tabular}{|c|c|c|c|c|}
\hline \multirow[t]{3}{*}{ Tipos de Drogas } & \multicolumn{4}{|c|}{ Faixa etária $(n=360)$ - Não informaram $(n=9)$} \\
\hline & \multicolumn{2}{|c|}{ Adulto $(n=323-87,5 \%)$} & \multicolumn{2}{|c|}{ Idoso $(n=37-10,0 \%)$} \\
\hline & $\mathrm{n}$ & $\%$ & $\mathrm{n}$ & $\%$ \\
\hline Tabaco & 145 & 44,9 & 14 & 37,8 \\
\hline Álcool & 250 & 77,4 & 31 & 83,8 \\
\hline Maconha & 93 & 28,8 & 2 & 5,4 \\
\hline Cocaína & 68 & 21,1 & 1 & 2,7 \\
\hline Crack & 122 & 37,8 & 0 & 0,0 \\
\hline \multirow[t]{3}{*}{ Tipos de Drogas } & \multicolumn{4}{|c|}{ Possui companheiro(a) $(n=341)$ - Não informaram $(n=28)$} \\
\hline & \multicolumn{2}{|c|}{$\operatorname{Sim}(n=118-32,0 \%)$} & \multicolumn{2}{|c|}{ Não $(n=141-38,2 \%)$} \\
\hline & $\mathrm{n}$ & $\%$ & $\mathrm{n}$ & $\%$ \\
\hline Tabaco & 55 & 44,0 & 70 & 56,0 \\
\hline Álcool & 95 & 45,7 & 113 & 54,3 \\
\hline Maconha & 27 & 38,0 & 44 & 62,0 \\
\hline Cocaína & 22 & 44,9 & 27 & 55,1 \\
\hline Crack & 31 & 36,0 & 55 & 64,0 \\
\hline
\end{tabular}

Fonte: Banco de dados da pesquisa sobre o perfil dos cientes dos CAPSad da região do Triângulo Mineiro (MG), 2016.

Nota: Categorias não são mutuamente exclusivas.

\section{Discussões}

Os usuários dos CAPSad do Triângulo Mineiro são predominantemente do sexo masculino, assim como revelado por outras investigações que avaliaram o perfil das populações atendidas nos CAPSad em diversos municípios e regiões do País ${ }^{7-14}$.

Com relação a faixa etária, estado civil e situação de trabalho, houve similaridade com outros estudos $8,9,12,13,15-18$.

O estudo de Faria e Schneider ${ }^{10}$, realizado no CAPSad do município de Blumenau (SC), apresentou as faixas etárias por segmentos, sendo que os usuários com mais de 34 anos eram a maioria em atendimento no serviço. Esse dado corrobora o de Freitas et al. ${ }^{16}$, que indica a incidência de maiores taxas a partir de 31 anos, referente ao CAPSad do município de Picos (PI). Monteiro ${ }^{24}$ define a faixa etária entre 19 e 59 anos (87,7\%) como prevalente no CAPSad de Teresina (PI). Os estudos que demonstraram essa variável, a partir da média de idade dos clientes, obtiveram 35,2 anos no município de Iguatu (CE) e 41,9 anos em Campo Grande (MS) $)^{8,19}$.

A maioria dos usuários dos CAPSad não possuía companheiro(a). Esse resultado também foi apresentado em outros estudos ${ }^{9,11,20}$. Pessoas que fazem uso abusivo de álcool e outras drogas têm maiores possibilidades de passarem por abandonos conjugais e separações. O isolamento social e a perda de vínculos familiares prejudicam a busca e a adesão ao tratamento. Já aqueles que ainda possuem vínculo familiar, principalmente filhos, têm maiores chances de iniciarem o tratamento e terem sucesso ${ }^{21}$.

$\mathrm{O}$ contexto familiar é um aspecto importante, que deve ser considerado na abordagem do uso de substâncias psicoativas. As relações familiares podem funcionar como um fator de proteção ou de risco para o comportamento de consumir drogas ${ }^{22}$. A família é um fator de proteção ao uso abusivo de drogas quando oferece ambiente doméstico harmônico, estável 
e seguro, com regras claras de conduta e envolvimento dos pais na vida dos filhos. Porém, pode ser considerada um risco quando o ambiente doméstico é desordenado, há consumo ou atitudes favoráveis com relação ao uso de drogas, pais que não proporcionam suporte e apoio social, ausência de monitoramento e expectativas altas e irrealistas entre os membros ${ }^{23}$.

No tocante à escolaridade, o estudo de Carvalho et al. ${ }^{8}$ demonstrou que $42,6 \%$ tinham entre quatro e sete anos de estudo. Horta et al. ${ }^{17}$ concluíram que $56,8 \%$ tinham até oito anos de escolaridade. Demais estudos apontam que: $71,0 \%$ dos usuários possuíam o ensino fundamental completo ${ }^{16}$; e 40,0\% ensino fundamental24. Esses resultados são semelhantes ao que foi verificado nesta pesquisa.

A baixa escolaridade da população investigada corrobora os resultados disponíveis na literatura, que sugere a relação entre a evasão escolar e o uso de drogas. Essa relação pode ter ocorrido não somente pelo uso de drogas, mas pelas consequências que o uso acarreta aos indivíduos, tais como dificuldades cognitivas e gerais de saúde 25 .

Com relação à situação de trabalho, a predominância de desemprego é a realidade de muitos estudos sobre o perfil dessa população ${ }^{\mathbf{1 8}}$. Neste estudo, a taxa de desemprego foi de $34,7 \%$, muito próxima da observada em estudo realizado por Monteiro et al. ${ }^{24}$, com taxa de $35,70 \%$. Em outras pesquisas que apresentaram a variável ocupação, houve predominância dos clientes que não possuíam nenhum tipo de ocupação, cujas porcentagens variaram de $51,5 \%$ a $87,3 \% 7,77,19,24,26$. Apesar de o desemprego ser uma questão de ordem estrutural e conjuntural na realidade da população estudada, ele torna-se mais significativo, visto que este representa um segmento vulnerável e marginalizado dentro do conjunto dos socialmente excluídos?

O consumo de álcool foi mais significativo entre os usuários que tinham ocupação, e o uso do crack entre as pessoas que não tinham. $\mathrm{O}$ uso habitual, abusivo e constante das drogas prejudica as relações sociais, familiares e de trabalho do indivíduo, afetando a qualidade de vida e a condição financeira. Tal situação causa uma desorganização da vida, levando ao absenteísmo, a dificuldades em cumprir as tarefas, à perda do emprego, ao descompromisso financeiro, à subvalorizacão da autoimagem e à manutenção de sentimentos negativos pelas perdas econômicas ${ }^{27}$.

A maioria dos clientes está em tratamento no CAPSad de 1 a 5 anos. Houve um percentual relevante de encaminhamentos ao CAPSad por outros serviços. Outros estudos também verificaram a demanda pelo atendimento do CAPSad, com prevalência dos encaminhamentos feitos por profissionais ou pelos serviços de saúde ${ }^{9,13}$. Esse resultado reforça a hipótese de que a atenção integral à saúde das pessoas que fazem uso nocivo de álcool e outras drogas está sendo constituída e articulada em rede, um dos pressupostos da Raps. Ao verificar que mais usuários estão sendo encaminhados pela rede pública, supõe-se que as unidades estejam mais integradas ao CAPSad, efetivando o funcionamento da Raps.

Os CAPSad oferecem as seguintes modalidades de tratamento: intensivo, semi-intensivo e não intensivo. O tratamento intensivo é direcionado às pessoas com grave sofrimento psíquico, que precisam de atenção contínua e diária. O semi-intensivo é oferecido às pessoas que necessitam de atenção direta da equipe, mas com melhor estruturação psíquica (o usuário pode ser atendido até 12 dias no mês). Já o tratamento não intensivo é oferecido às pessoas que não necessitam de suporte contínuo, limitando-se o seu atendimento a até três dias no mês. As atividades consistem em atendimento individual, em grupo, oficinas terapêuticas, visitas domiciliares, repouso e desintoxicação ambulatorial ${ }^{3}$. A modalidade mais utilizada foi a semi-intensiva, conforme apresentado em outros estudos, 7,13,15,16.

Os resultados desta pesquisa evidenciaram, também, que a maioria dos usuários dos CAPSad, onde o estudo se desenvolveu, já esteve internada em hospitais ou clínicas por uso de substâncias psicoativas. Dos três CAPSad participantes da pesquisa, apesar de 
dois serem registrados como CAPSad III 24 horas, apenas um estava, efetivamente, funcionando nesse formato, que disponibiliza o cuidado contínuo, fundamental para o acompanhamento das situações de maior complexidade e atenção à crise no território, aprofundando as ações substitutivas ao modelo de internação em hospitais e/ou clínicas especializadas. A carência dessa modalidade de Caps fragiliza as ações de retaguarda e de garantia de cuidados contínuos e de gestão da crise no território de forma regionalizada. Trata-se, deste modo, de um ponto crítico para responder de forma substitutiva às necessidades de internação.

O CAPSad III não tem por finalidade oferecer estrutura hospitalar, mas ter recursos para atuar de forma articulada na Raps quando for necessário o cuidado intensivo, de 24 horas, do usuário do serviço. A implementação desse dispositivo como uma forma de reorganização da rede de atenção tem mostrado resultados satisfatórios, como a redução das internações e do tempo de permanência dos usuários em hospitais psiquiátricos, e também dos casos de emergências relacionadas ao uso de álcool e outras drogas que chegam às Unidades de Pronto Atendimento ${ }^{\mathbf{2 8}}$.

A maioria dos clientes iniciou o uso de substâncias psicoativas na faixa etária entre 12 e 15 anos. Esse resultado está de acordo com outros estudos que abordaram essa variável14,17,29,30. Os levantamentos epidemiológicos sobre o consumo de álcool e outras drogas no mundo e no Brasil mostram que é nessa fase do desenvolvimento que se inicia o uso de substâncias nocivas à saúde ${ }^{31-35}$. O período da adolescência é marcado por intensas mudanças e adaptações que o indivíduo vivencia na transição para a fase adulta. É um período considerado crítico no que se refere ao desenvolvimento de competências pessoais e interpessoais, aquisição de habilidades e tomada de decisões. Essa fase do desenvolvimento constitui um período crucial no ciclo vital para o início do uso de drogas, seja como mera experimentação, seja como consumo ocasional, indevido ou abusivo ${ }^{22}$. Apenas um dos três CAPSad investigados realizava atendimentos direcionados a essa população.

O conceito de redução de danos é preconizado pela Política do Ministério da Saúde para a Atenção Integral a Usuários de Álcool e outras Drogas e propõe diretrizes que colocam o usuário no papel de protagonista de sua vida e do seu tratamento. O Ministério da Saúde compreende, a partir dessa política, que a abstinência não é o único caminho a ser percorrido nem, necessariamente, deva ser o ponto de partida no tratamento. Considerar as singularidades de cada sujeito é fundamental para as práticas de saúde, que devem considerar a diversidade e envolver a participação e o engajamento da pessoa em tratamento. A redução de danos é uma prática onde as estratégias são construídas com os usuários, com foco na liberdade, na corresponsabilidade e na capacidade do usuário de fazer suas próprias escolhas $3,36,37$.

Este estudo investigou, também, os tipos de drogas consumidas pelos usuários dos CAPSad durante a vida e no momento do acolhimento no serviço. A substância mais consumida ao longo da vida e no momento de acolhimento foi o álcool, seguido pelo tabaco. Esses dados encontram ressonância em estudos e levantamentos feitos na população geral e na população em tratamento decorrente do uso de substâncias psicoativas 7,9,10,16.

Em 2015, as mortes relacionadas ao consumo abusivo de álcool e dependência de álcool no Brasil corresponderam a 93,5\% do total de óbitos por causa básica atribuída ao uso de substâncias psicoativas ${ }^{38}$. O uso abusivo do álcool é um dos fatores de risco de maior impacto para a morbidade, mortalidade e incapacidades em todo o mundo, e, segundo dados da OMS, está relacionado a 3,3 milhões de mortes a cada ano. Dessa forma, quase $6 \%$ de todas as mortes em todo o mundo são atribuídas total ou parcialmente ao álcool. No Brasil, o álcool esteve associado a $63 \% \mathrm{e}$ $60 \%$ dos índices de cirrose hepática e a $18 \%$ e $5 \%$ dos acidentes de trânsito entre homens e mulheres em 2012, respectivamente ${ }^{39}$. 
Entretanto, existem os interesses econômicos sobre o consumo de bebidas alcoólicas no Brasil, que estão relacionados, também, com o mercado econômico, já que representa uma importante receita. De acordo com os dados da Pesquisa de Orçamentos Familiares (POF), realizada pelo Instituto Brasileiro de Geografia e Estatística IBGE (2008-2009), que investigou o consumo de alimentos e outros produtos a partir dos gastos familiares, houve aumento no consumo, nos últimos 40 anos, num ritmo médio de $2,5 \%$ ao ano. As despesas com bebidas alcoólicas têm maior participação nos gastos familiares mensais à medida que aumenta a renda familiar. Também é maior nas áreas urbanas, com maior participação relativa de outros tipos de bebidas diferentes de cervejas e chopes ${ }^{40}$.

As estratégias para a diminuição do consumo abusivo de álcool são fundamentais para minimizar os impactos na saúde, nos âmbitos individual e coletivo, incluindo a redução da sobrecarga no sistema de saúde devido a agravos relacionados ao consumo abusivo de álcool. Entre as medidas recomendadas pela OMS, o aumento dos preços das bebidas alcoólicas é uma forma efetiva para a redução do consumo abusivo de álcool, o controle rígido sobre o acesso a bebidas alcoólicas, incluindo a restrição do horário para a venda e maior regulação e restrição dos pontos de venda ${ }^{38}$.

Uma limitação do estudo se dá com relação aos dados coletados a partir de informações produzidas pelos próprios serviços e registradas nos prontuários. Houve um índice de dados não preenchidos nos prontuários dos clientes dos três CAPSad. O registro das informações das instituições públicas de saúde deve ser feito corretamente, para possibilitar as análises epidemiológicas e viabilizar fontes fidedignas para pesquisas científicas.

\section{Conclusões}

O conhecimento do perfil das pessoas atendidas nos CAPSad permite o desenvolvimento de intervenções focadas na realidade e nas demandas dessa população. A atenção psicossocial realizada nos CAPSad deve ser constantemente avaliada em um contínuo processo de pensar as práticas e recriá-las, a fim de aprimorar as ações, ampliando as atividades clínicas em busca de atender às necessidades.

O perfil da maioria dos usuários dos CAPSad onde o estudo foi realizado foi de homens, entre 41 e 60 anos, sem companheiro(a) e desempregados. Esse panorama reforça a necessidade de resgatar a autonomia desses sujeitos, proporcionando sua reinserção na comunidade, através do trabalho com valor social, da busca dos direitos de cidadania a partir de uma assistência especializada, individualizada e que traga melhoria da qualidade de vida dessa população e de seus familiares. As redes sociais de apoio, a intersetorialidade e a articulação da Raps devem ser a base de sustentação para que essas questões passem do discurso e se concretizem na prática.

Procedimentos que estejam em consonância com os preceitos da Raps, com práticas intersetoriais disponíveis na comunidade, desenvolvimento de projetos de promoção da saúde física e mental, programas de redução de danos, elaboração de programas de cooperativas sociais de trabalho e inclusão social, podem ser importantes estratégias para a diminuição do consumo do álcool e para minimizar os impactos na saúde nos âmbitos individual e coletivo.

\section{Colaboradores}

Trevisan ER (0000-0002-3015-1630)* contribuiu para a concepção, o planejamento, a análise e a interpretação dos dados; revisão crítica do conteúdo; e aprovação da versão final do manuscrito. Castro SS (0000-0002-00057555)* contribuiu para a análise e a interpretação dos dados; revisão crítica do conteúdo e a aprovação da versão final do manuscrito. 


\section{Referências}

1. Amarante P, Nunes MO. A reforma psiquiátrica no SUS e a luta por uma sociedade sem manicômios. Ciênc. Saúde Colet. 2018; 23:2067-2074.

2. Trevisan ER, Castro SS. Aspectos psicossociais do trabalho em Saúde Mental: uma revisão integrativa. SMAD Rev Eletrônica Saúde Ment. Álcool e Drog. 2016; 12(3):188-197.

3. Brasil. Ministério da saúde. A política do Ministério da Saúde para a atenção integral a usuários de álcool e outras drogas [internet]. Brasília, DF: Ministério da Saúde; 2003. [acesso em 2019 jul 4]. Disponível em: http://bvsms.saude.gov.br/bvs/publicacoes/pns_alcool_drogas.pdf.

4. Oliveira MAF, Cestari TY, Pereira MO, et al. Processos de avaliação de serviços de saúde mental: uma revisão integrativa da literatura. Saúde debate [internet]. 2014 [acesso em 2015 maio 19]; 38(101). Disponível em: http://www.scielo.br/pdf/sdeb/v38n101/01031104-sdeb-38-101-0368.pdf.

5. World Health Organization. World drug report 2016. Genebra: United Nations Pubns; 2016.

6. Claro HG, Oliveira MAF, Bourdreaux JT, et al. Drug use, mental health and problems related to crime and violence: cross-sectional study. Rev. Latino-Am. Enfermagem. 2015; 23(6):1173-1180.

7. Almeida RA, Anjos UU, Vianna RP T, et al. Profile of users of psychoactive substances in João Pessoa. Saúde debate. 2014; 38(102):526-538.

8. Carvalho MDA, Silva HO, Rodrigues LV. Perfil epidemiológico dos usuários da Rede de Saúde Mental do Município de Iguatu, CE. SMAD Rev Eletrônica Saúde Ment. Álcool e Drog. 2010; 6(2):337-349.

Constantino P, Batista LSS. Batista M. Perfil dos usuários de substâncias psicoativas do caps ad em 2000 e 2009, Campos dos Goytacazes, RJ. Persp. online: biol \& saúde. 2012; 7(2):23-38.
10. Faria JG, Schneider DR. O perfil dos usuários do CAPSad-Blumenau e as políticas públicas em saúde mental. Psicol. soc. 2009; 21(3):324-333.

11. Marini M. Preditores de adesão ao projeto terapêutico em centro de atenção psicossocial álcool e outras drogas [dissertação] [internet]. Porto Alegre: Universidade Federal do Rio Grande do Norte, Escola de Enfermagem; 2011 [acesso em 2015 mar 31]. Disponível em: http://www.lume.ufrgs.br/handle/10183/36963.

12. Pelisoli CL, Moreira ÂK. Epidemiological characterization of the users of Casa Aberta, a psychosocial attention center. Rev. psiquiatr. Rio Gd. Sul. 2005; 27(3):270-277.

13. Pelisoli CL, Moreira ÂK. Avaliação de um centro de atenção psicossocial por meio do perfil de seus usuários. Mental. 2007; 5(8):61-75.

14. Pereira MO, Souza JM, Costa ÂM, et al. Perfil dos usuários de serviços de saúde mental do município de Lorena-São Paulo. Acta Paul. Enferm. 2012; 25(1):48-54.

15. Freitas AA, Souza RC. Caracterização clínica e sociodemográfica dos usuários de um centro de atenção psicossocial (CAPS). Rev. baiana saúde pública. 2010; 34(3):100-233.

16. Freitas RM, Silva HRR, Araújo DS. Resultados do acompanhamento dos usuários do Centro de Atenção Psicossocial - Álcool e Drogas (Caps-AD). SMAD Rev Eletrônica Saúde Ment. Álcool e Drog. 2012; 8(2):5663.

17. Horta RL, Horta BL, Rosset AP. Perfil dos usuários de crack que buscam atendimento em Centros de Atenção Psicossocial. Cad. Saúde Pública. 2011; 27(11):2263-2270.

18. Jorge JS, Corradi-Webster CM. Consultório de Rua: contribuições e desafios de uma prática em construção. Sau. \& Transf. Soc. 2012; 3(1):39-48. 
19. Peixoto C, Prado CHO, Rodrigues CP, et al. Impact of clinical and socio-demographic profiles in treatment adherence of patients attending a day hospital for alcohol and drug abuse. J. bras. psiquiatr. 2010; 59(4):317-321.

20. Rocha WS, Alves ERP, Vieira KFL, et al. Concepções dos usuários de crack sobre os fatores que influenciam o uso e a dependência. SMAD Ver. Eletrônica Saúde Ment. Álcool e Drog. 2015; 11(3):129-135.

21. Almeida CS, Luis MAV. Características sociodemográficas e padrão de uso de crack e outras drogas em um Caps. Rev. enferm. UFPE on line. 2017; 11(4):17161723.

22. Schenker M, Minayo MCS. Risk and protective factors and drug use among adolescence. Ciênc. Saúde Colet. 2005; 10(3):707-17.

23. Ribeiro M, Dualibi L. Avaliação de fatores de proteção e de risco. In: Laranjeira R, organizador. O tratamento do usuário de crack. São Paulo: Casa Leitura Médica; 2010. p. 175-86.

24. Monteiro CFS, Fé LCM, Moreira MAC, et al. Perfil sociodemográfico e adesão ao tratamento de dependentes de álcool em CAPS-ad do Piauí. Esc. Anna Nery Rev. Enferm. 2011; 15(1):90-95.

25. Araujo NB, Marcon SR, Silva NG. Clinical and sociodemographic profile of adolescents who stayed and did not stay in treatment at CAPSad Cuiabá/MT. J. bras. psiquiatr. 2012; 61(4):227-234.

26. Hess ARB, Almeida RMM, Moraes AL. Comorbidades psiquiátricas em dependentes químicos em abstinência em ambiente protegido. Estud. Psicol. 2012; 17(1):171-178.

27. Nimtz MA, Tavares AMF, Maftum MA, et al. Impactos legais e no trabalho na vida do dependente químico. SMAD Rev. Eletrônica Saúde Ment. Álcool e Drog. 2016; 12(2):68-74.

28. Boska GA, Oliveira MAF, Claro HG, et al. Leitos em centro de atenção psicossocial álcool e drogas: análi- se e caracterização. Rev. bras. enferm. 2018; 71:22512257.

29. Figlie NB, Bordin S, Laranjeira R. Aconselhamento em Dependência Química. 2. ed. São Paulo: Roca; 2010.

30. Lopes AP, Rezende MM. Consumo de substâncias psicoativas em estudantes do ensino médio. Psicol. teor. prát. 2014; 16(2):29-40.

31. Domingos NAM, Domingos JCV. Levantamento sobre o uso de álcool e drogas em universitários. Rev. bras. ter. cogn. 2005; 1(1):75-82.

32. Galduróz JCF, Noto AR, Nappo SA, et al. Uso de drogas psicotrópicas no Brasil: pesquisa domiciliar envolvendo as 107 maiores cidades do país-2001. Rev. Lat. Am. Enfermagem. 2005; 13(1):888-895.

33. Laranjeira R, Madruga CS, Pinsky I, et al. II levantamento nacional de álcool e drogas [internet]. São Paulo: Instituto Nacional de Ciência e Tecnologia para Políticas Públicas do Álcool e Outras Drogas; 2013. [acesso em 2019 jun 30]. Disponível em: http://inpad. org.br/wp-content/uploads/2013/04/LENAD_PressRelease_Alcohol_RVW.pdf.

34. Pratta EMM, Santos MA. Levantamento dos motivos e dos responsáveis pelo primeiro contato de adolescentes do ensino médio com substâncias psicoativas. SMAD Rev. Eletrônica Saúde Ment. Álcool E Drog. 2006; 2(2):1-17.

35. Silveira DX. Reflexões sobre a prevenção do uso indevido de drogas. In: Silveira DX, Niel M, organizadores. Drogas e redução de danos: uma cartilha para profissionais de saúde [internet]. São Paulo; 2008 [acesso em 2015 ago 14]. Disponível em: http://www.researchgate.net/profile/Dartiu_Silveira/publication/230824010_Drogas_e_reduo_de_ danos_uma_cartilha_para_profissionais_de_sade/ links/09e41509165f4641c3000000.pdf\#page=7.

36. Brasil. Ministério da Saúde, Secretaria de Atenção à Saúde. Reforma psiquiátrica e política de saúde mental no Brasil. Brasília, DF: OPAS; 2005. [acesso em 2019 
jun 30]. Disponível em: http://bvsms.saude.gov.br/ bvs/publicacoes/Relatorio15_anos_Caracas.pdf.

37. Calassa GDB, Penso MA, Freitas LG. Redução de danos na visão dos profisssionais que atuam no CAPS AD II do Distrito Federal. Psicol. pesq. 2015; 9(2):177-187.

38. Munhoz TN, Santos IS, Nunes BP, et al. Tendências de consumo abusivo de álcool nas capitais brasileiras entre os anos de 2006 a 2013: análise das informações do Vigitel. Cad. Saúde Pública [internet]. 2017 [acesso em 2017 out 24]; 33(7):1-11. Disponivel em: http://www.scielo.br/scielo.php?script=sci_arttext\&pid=S0102-311X20 17000705011\&lng=pt\&tlng=pt.
39. World Health Organization. Global status report on alcohol and health, 2014. Geneva: World Health Organization; 2014.

40. Yamamoto CH. A demanda por bebidas alcoólicas no Brasil: 2008/2009 [dissertação]. São Paulo: Fundação Getúlio Vargas; 2011.

Recebido em 24/09/2018

Aprovado em 17/02/2019

Conflito de interesses: inexistente

Suporte financeiro: Fundação de Apoio à Pesquisa de Minas

Gerais (Fapemig) 